\title{
Fé de erratas
}

En la publicación del volumen 46, número 4, página 443 de la Rev Chil Nutr dice:

Claudia Ramírez', Johana Quintero', Isabel Jamioi', Santiago Guerra'.

1. Grupo de Investigación en Salud Familiar y Comunitaria, Programa de Nutrición y Dietética, Facultad de Ciencias de la Salud, Corporación Universitaria Remington. Calle 51 N 51 -27, Medellín, Colombia.

Y debe de decir:

Claudia Ramírez1, Johana Quintero², Isabel Jamioi², Santiago Guerra².

1. Grupo de Investigación en Salud Familiar y Comunitaria, Programa de Nutrición y Dietética, Facultad de Ciencias de la Salud, Corporación Universitaria Remington. Calle 51 Nº51-27, Medellín, Colombia.

2. Escuela de Nutrición y Dietética. Universidad de Antioquia. Ca rrera $75 N^{\circ}$ 65-87. Medellín. Colombia. 